\title{
Caracterización de la gestión universitaria dentro de la Universidad Laica Eloy Alfaro de Manabí (ULEAM), Ecuador
}

\begin{abstract}
RESUMEN
Este artículo se centra en uno de los problemas más trascendentales de la educación superior en el Ecuador: la gestión universitaria. Su definición es el único elemento proyectado con perspectiva académica; pues se ha olvidado procurar el accionar administrativo y rige la escasez de información sobre sus componentes, sin los cuales no es posible examinarlo ni caracterizarlo. Por ello, en este estudio se hace un análisis y una descripción de la gestión de la Universidad Laica Eloy Alfaro de Manabí (ULEAM) a través de la aplicación de la "Dimensión II. Misión y proyecto institucional. Gobierno y gestión", que integra el instrumento usado por la Comisión Nacional de Evaluación y Acreditación Universitaria (CONEAU) de Argentina en sus procesos de evaluación. Esta herramienta ha sido adaptada a la encuesta con escalamiento Likert, con el fin de recolectar datos y aplicarla al personal titular docente y no docente. Finalmente, los resultados muestran una seria debilidad en los factores gobierno, gestión y gobernabilidad.
\end{abstract}

Palabras-claves: Organización; gestión universitaria; educación superior; gobernabilidad.

\section{INTRODUCCIÓN}

Una organización es un grupo de personas con estructura establecida que, en conjunto y al pie de sus características, cumple una serie de objetivos planteados en común (Carbajal, 2010). Por tanto, las universidades también son organizaciones con estructuras, responsabilidades y demandas, en las que tiene relevancia la acción categórica de sus miembros y la guía de un mando (Grisales y Monroy, 2011). Si una organización mantiene una relación recíproca entre personas que coordinan medios, procesos y un elemento humano con ordenamiento dinámico enfocado al logro de propósitos, entonces una apropiada gestión universitaria favorecerá la eficiencia de los procesos académicos, el aseguramiento de la calidad, y la guiará a la concesión de la acreditación universitaria. En consecuencia, se permitirá alinear la actuación de cada persona y unidad de su estructura a la política y las estrategias institucionales, con el fin de que pueda sustentarse en normas y procedimientos claros que eviten funciones repetitivas o ambiguas, y que cuente con recursos suficientes y con competencias pertinentes.

Al proceso de administrar las actividades de una organización con alto grado de responsabilidad y otras características anexas, se denomina gestión institucional; sin embargo, cuando la institución sujeta a esta acción es de educación superior, se trata de una gestión universitaria. La gestión es un conjunto de tareas realizadas para la obtención de un producto de naturaleza administrativa, de modo que el término es más usado en actividades programadas y planificadas. Con la inclusión del término liderazgo, se amplía su conceptualización y ejercicio, además de otorgarle mayor alcance y no limitarla al cumplimiento de las actividades relacionadas con los cuatro procesos básicos de la administración (planeación, organización, dirección y control). Uno de los papeles importantes de la gestión universitaria es el manejo de la academia, a la cual se debe integrar la ciencia, la

1 Magíster en Gerencia Educativa por la Universidad Estatal del Sur de Manabí (Ecuador), ha estudiado Ingeniera Industrial en la Universidad Laica Eloy Alfaro de Manabí (Ecuador) y es docente titular de la misma institución. Manabí-Ecuador.

E-mail: jahaira.quijije@uleam.edu.ec

2 Magíster en Dirección de Comunicación Empresarial e Institucional, por la Universidad de las Américas (Ecuador), y licenciada en Comunicación Social, con mención en Comunicación Organizacional y Relaciones Públicas, por la Universidad Laica Eloy Alfaro de Manabi (Ecuador). Es docente de la Unidad Educativa del Milenio Dra. Guadalupe Larriva (Ecuador). Manabí-Ecuador.

E-mail: kerly.quijije@educacion.gob.ec 
administración y la cultura para su servicio (Cejas y Robaina, 2012). Por ese motivo, es indispensable la relación entre la administración y la gestión, ya que ello contribuirá con el realce de esta última.

La Comisión Nacional de Evaluación y Acreditación Universitaria (CONEAU) (1998) de Argentina dio un paso más, pues caracterizó a la gestión universitaria desde la perspectiva que se tenía hasta entonces sobre la misma; es decir, entendida a partir de la estructuración de las funciones y del manejo de recursos disponibles para el efecto. Se debe recordar que la universidad no solo debe ser entendida como una organización, sino también como una entidad de amplias y dinámicas extensiones, en la que habrán de considerarse aquellas que la integren desde una perspectiva humana, producto de una educación formal (Mujica y Pérez, 2009). De esta forma, la creación de la institución universitaria ha pasado por un proceso evolutivo para poder conceptualizar a la universidad y, por ende, a su gestión, sin olvidar tomar en cuenta a su historia (Cejas y Robaina, 2012; Álvarez, Cejas, Robaina y Alonso, 2013). En la actualidad, la universidad moderna está obligada a una revisión constante de su modelo de gestión con el objetivo de lograr redireccionar sus actividades para que estas se articulen mejor frente a los nuevos procesos que demanda la educación (Garbanzo y Romero, 2013).

El estudio forma parte de una investigación que relaciona dos temas referentes a la educación superior: la gestión universitaria, definida como el proceso de planificar, organizar, dirigir y controlar; es decir, aplicar los pilares conocidos popularmente de la administración, pero con alto grado de diligencia, responsabilidad y eficiencia; y el clima organizacional, conocido como la percepción de los trabajadores de una organización hacia su entorno laboral y que tiene repercusión en su productividad. Además, el tema ofrece mayor entendimiento sobre la divergencia en las definiciones entre administración y gestión, pues busca introducir al lector y, en particular, a la comunidad universitaria en el conocimiento del estado de la gestión institucional en la ULEAM en Ecuador.
Se guarda justificación desde el enfoque teórico, donde se analizan los componentes de la gestión universitaria de la ULEAM y se los caracteriza. Asimismo, ello contribuye con su conceptualización como institución; encamina su sistema administrativo y educativo; favorece la satisfacción de sus usuarios, las necesidades de su entorno laboral; y establece un precedente para que se incorpore el enfoque organizacional a sus procesos. No obstante, una de las limitaciones más importantes se encontró durante la recolección de datos, en la que se observó cierto grado de temor a contestar las preguntas del cuestionario, específicamente en los indicadores sobre gobierno y gobernabilidad.

\section{METODOLOGÍA}

El estudio tiene un alcance de tipo exploratorio, dado que la gestión en la universidad es instrumental y se expone a sí misma como objeto de estudio. También es de tipo descriptivo, ya que se recopila información sobre las variables para poder establecer sus características y facilitar la discriminación entre factores de interés. De igual manera, es de tipo no experimental, pues no hay manipulación de variables (Hernández, Fernández y Baptista, 2014).

Por otro lado, la población de 928 individuos lo conforman el personal titular académico y no académico que labora en la ULEAM. Asimismo, se determinó el tamaño de la muestra con la ecuación para una población finita, con un nivel de confianza del $95 \%$ y un error muestral del $5 \%$; la técnica del muestreo es probabilística y estratificada (Hernández, Fernández y Baptista, 2014). De esta forma, se obtuvieron 272 encuestas válidas, como se muestra en la Tabla 1.

La técnica usada para recabar datos fue la encuesta escrita, la cual es, como la define Arias (2012), un cuestionario de preguntas cerradas. Se usó la "Dimensión II. Misión y proyecto institucional. Gobierno y gestión", que conforma el conjunto de dimensiones que integran el instrumento de Evaluación institucional: criterios y procedimientos para la evaluación externa, el cual es resultado del ajuste efectuado al documento Lineamientos para la eva-

Tabla 1. Datos de la muestra.

\begin{tabular}{|l|c|c|}
\hline \multicolumn{1}{|c|}{ Estratos } & N. ${ }^{\circ}$ de encuestados & Porcentaje \\
\hline Personal académico: docente & 215 & $79 \%$ \\
\hline Personal no académico: administrativo y servicio & 57 & $21 \%$ \\
\hline Total & 272 & $100 \%$ \\
\hline
\end{tabular}

Fuente: Elaboración propia. 
luación institucional. Originalmente, constaba solo de tres variables, a las que se le añadió la gobernabilidad, propuesta en la Evaluación de la gestión universitaria, con la finalidad de conseguir mayor información sobre los órganos de gobierno. La Tabla 2 detalla el instrumento final.

Al cuestionario se le adaptó un escalamiento Likert, vigente y popular en estudios que miden percepciones, con categorías del 1 al 5 , donde 1 significa "en total desacuerdo" y 5 , "en total acuerdo".

En la validez de contenido se efectuó lo siguiente:

- Una revisión bibliográfica especializada desde 2009 hasta la presente fecha.

- Una comparación entre administración y gestión a partir de ocho autores que datan de 2000, así como desde 2009 hasta la actualidad.

- Un análisis de cuatro criterios con socialización de 22 dimensiones.

- Una revisión de ocho modelos de medición, con 36 variables y más de 465 indicadores, los cuales incluyen el tipo de gestión empresarial, educacional y uno europeo.

- Una revisión de la información contenida en los Coloquios Internacionales de Gestión Universitaria (CIGU) de versiones anteriores a partir del 2000, evento organizado en asociación con la Universidad Federal de Santa Catarina (UFSC), el Instituto de Investigaciones y Estudios en Administración Universitaria (INPEAU), la Universidad Nacional de Mar del Plata (UNMDP) y la Asociación de Especialistas en Gestión de la Educación Superior (AEGES) en Argentina.

- Estudios sobre la práctica de la gestión y el gestor.

- Una revisión de ocho criterios referentes a las competencias de la gestión universitaria.

- Una adaptación del material original con bibliografía especializada.

El estadístico para medir la consistencia interna fue el alfa de Cronbach. Además, se usó el programa estadístico SPSS para el procesamiento de datos.

\section{RESULTADOS}

En estudios actitudinales, la media puede ser afectada por valores atípicos que la desestabilicen; por tanto, el análisis de los resultados se basa exhaustivamente en los estadísticos mediana y moda, recomendados por Orlandoni (2010) para escalas de medición con datos ordinales por su elevada propiedad representativa de la muestra (Salazar y Castillo, 2017).

En la Tabla 3 se muestran estos parámetros para la primera variable.

Tabla 2. Instrumento para la caracterización de la gestión universitaria.

\begin{tabular}{|l|l|l|c|}
\hline \multicolumn{1}{|c|}{ Modelo } & \multicolumn{1}{|c|}{ Autor } & \multicolumn{1}{|c|}{ Variables } & Indicadores \\
\hline $\begin{array}{l}\text { Dimensión II. Misión y Proyecto institucional. } \\
\text { Gobierno y gestión (2016). }\end{array}$ & $\begin{array}{l}\text { Comisión Nacional de Evaluación y } \\
\text { Acreditación Universitaria (CONEAU) }\end{array}$ & $\begin{array}{l}\text { 1. Misión y proyecto institucional } \\
\text { 2. Gobierno y gestión } \\
\text { 3. Gobernabilidad } \\
\text { 4. Gestión económico-financiera }\end{array}$ & 44 \\
\hline
\end{tabular}

Fuente: Elaboración propia a partir de la información extraída de la CONEAU (1998), la CONEAU (2016) y Martínez (2000).

Tabla 3. Percepción sobre los indicadores. Variable: Misión y proyecto institucional.

\begin{tabular}{|c|c|c|c|c|c|}
\hline \multicolumn{2}{|r|}{ Indicador } & \multirow{2}{*}{$\begin{array}{c}\text { Media } \\
3,81\end{array}$} & \multirow{2}{*}{$\begin{array}{c}\text { Desviación } \\
\text { estándar }\end{array}$} & \multirow{2}{*}{$\begin{array}{c}\text { Mediana } \\
4\end{array}$} & \multirow{2}{*}{$\frac{\text { Moda }}{5}$} \\
\hline 1 & Está claramente definida la misión institucional en los documentos oficiales. & & & & \\
\hline 2 & Están claramente definidos los objetivos institucionales en los documentos oficiales. & 3,81 & 1,27 & 4 & 5 \\
\hline 3 & $\begin{array}{l}\text { El plan de desarrollo institucional y/o plan estratégico se articula con la misión y los } \\
\text { objetivos institucionales. }\end{array}$ & 3,75 & 1,18 & 4 & 4 \\
\hline 4 & La misión es conocida y aceptada por la comunidad universitaria. & 3,74 & 1,14 & 4 & 4 \\
\hline 5 & Los objetivos institucionales son conocidos y aceptados por la comunidad universitaria. & 3,61 & 1,13 & 4 & 4 \\
\hline 6 & $\begin{array}{l}\text { La misión es adecuadamente expresada por los actores principales y en todas las } \\
\text { actividades académicas. }\end{array}$ & 3,77 & 1,08 & 4 & 4 \\
\hline 7 & $\begin{array}{l}\text { Los objetivos institucionales son adecuadamente expresados por los actores principa- } \\
\text { les y en todas las actividades académicas. }\end{array}$ & 3,73 & 1,12 & 4 & 4 \\
\hline
\end{tabular}

Fuente: Elaboración propia. 
Luego, en la Figura 1 se muestran los valores promedios observados para cada ítem.

Como consecuencia, la muestra indicó estar "en parte de acuerdo" con los indicadores de la variable "Misión y proyecto institucional". El menor valor promedio fue para el indicador 5 , el cual refiere a los objetivos institucionales. La categoría 4 fue la que más se repitió (moda) con "en parte de acuerdo" en la mayoría de los indicadores. Además, el 50\% de los individuos está por encima del valor de 4 y el restante $50 \%$ se sitúa por debajo de este valor (mediana).

La Tabla 4 muestra los resultados para la segunda variable.

\section{Variable: Misión y proyecto institucional}

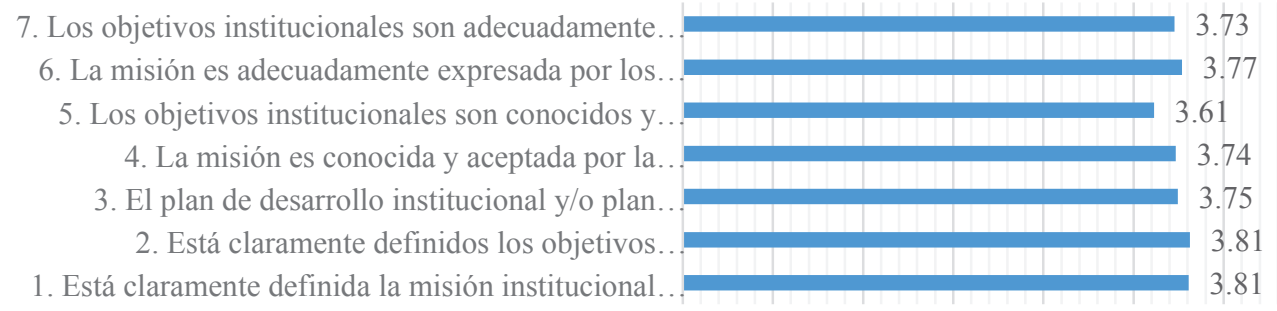

$$
\begin{array}{lllllllll}
1.00 & 1.50 & 2.00 & 2.50 & 3.00 & 3.50 & 4.00 & 4.50 & 5.00
\end{array}
$$

Figura 1. Media de la percepción por indicador.

Fuente: Elaboración propia.

Tabla 4. Percepción sobre los indicadores. Variable: Gobierno y gestión.

\begin{tabular}{|c|c|c|c|c|c|}
\hline \multicolumn{2}{|r|}{ Indicador } & \multirow{2}{*}{\begin{tabular}{|c|} 
Media \\
3,14 \\
\end{tabular}} & \multirow{2}{*}{\begin{tabular}{|c|}
$\begin{array}{c}\text { Desviación } \\
\text { estándar }\end{array}$ \\
1,32 \\
\end{tabular}} & \multirow{2}{*}{\begin{tabular}{|c|} 
Mediana \\
3 \\
\end{tabular}} & \multirow{2}{*}{$\begin{array}{c}\text { Moda } \\
4\end{array}$} \\
\hline 8 & $\begin{array}{l}\text { Los procesos decisorios para la adopción de políticas guardan autonomía académica e } \\
\text { institucional. }\end{array}$ & & & & \\
\hline 9 & La comunidad universitaria tiene participación en los procesos decisorios. & 2,88 & 1,35 & 3 & 4 \\
\hline 10 & Existe información disponible para la toma de decisiones. & 2,95 & 1,45 & 3 & 4 \\
\hline 11 & $\begin{array}{l}\text { Existen mecanismos para captar la opinión de alumnos, docentes y administrativos } \\
\text { sobre aspectos decisorios que le son pertinentes. }\end{array}$ & 2,95 & 1,34 & 3 & 4 \\
\hline 12 & $\begin{array}{l}\text { Existe un conocimiento difundido y entendido de la actual estructura organizacional } \\
\text { universitaria. }\end{array}$ & 2,85 & 1,33 & 3 & 4 \\
\hline 13 & La estructura organizacional universitaria se ajusta a la normativa institucional. & 3,05 & 1,29 & 3 & 4 \\
\hline 14 & La organización del gobierno universitario se ajusta a la normativa institucional. & 3,06 & 1,31 & 3 & 4 \\
\hline 15 & La comunidad universitaria tiene participación en la revisión de la normatividad institucional. & 2,99 & 1,33 & 3 & 2 \\
\hline 16 & $\begin{array}{l}\text { Existen resultados correctivos concretos como consecuencia de la aplicación de los } \\
\text { sistemas de control de gestión. }\end{array}$ & 3,24 & 1,17 & 3 & 4 \\
\hline 17 & El personal técnico para las necesidades de gestión es suficiente. & 3,11 & 1,17 & 3 & 4 \\
\hline 18 & El personal administrativo para las necesidades de gestión es suficiente. & 3,11 & 1,32 & 3 & 4 \\
\hline 19 & $\begin{array}{l}\text { La infraestructura permite un desarrollo adecuado de las funciones de docencia, investi- } \\
\text { gación y extensión. }\end{array}$ & 2,47 & 1,29 & 2 & 1 \\
\hline 20 & $\begin{array}{l}\text { El equipamiento permite un desarrollo adecuado de las funciones de docencia, investi- } \\
\text { gación y extensión. }\end{array}$ & 2,59 & 1,29 & 2 & 2 \\
\hline 21 & $\begin{array}{l}\text { Los sistemas informáticos facilitan el acceso a la información para la toma de decisiones } \\
\text { y el control de gestión. }\end{array}$ & 3,22 & 1,18 & 3 & 4 \\
\hline 22 & $\begin{array}{l}\text { Los sistemas administrativos (recursos humanos; compras, adquisiciones y contratacio- } \\
\text { nes; pagos, tesorería, liquidaciones y viáticos; gestión contable, financiera y patrimonial; } \\
\text { etc.) son efectivos. }\end{array}$ & 2,60 & 1,25 & 2 & 2 \\
\hline 23 & $\begin{array}{l}\text { La institución es capaz de formular diagnósticos y generar políticas en el marco de un } \\
\text { planeamiento estratégico. }\end{array}$ & 3,34 & 1,10 & 4 & 4 \\
\hline 24 & $\begin{array}{l}\text { Se han tomado en cuenta las recomendaciones de las evaluaciones institucionales pre- } \\
\text { vias, en la formulación de los planes de desarrollo institucional y/o plan estratégico. }\end{array}$ & 3,29 & 1,14 & 3 & 4 \\
\hline 25 & $\begin{array}{l}\text { Se han tomado en cuenta los diagnósticos y recomendaciones en el proceso de autoe- } \\
\text { valuación institucional de las evaluaciones institucionales previas. }\end{array}$ & 3,36 & 1,12 & 4 & 4 \\
\hline 26 & La institución cuenta con planes de mejora para las distintas funciones. & 3,46 & 1,18 & 4 & 4 \\
\hline 27 & La institución cuenta con recursos para la planificación de las distintas funciones. & 3,25 & 1,17 & 3 & 4 \\
\hline
\end{tabular}

Fuente: Elaboración propia. 
Seguidamente, la Figura 2 muestra la media obtenida para cada indicador de la variable. En la variable "Gobierno y gestión", los encuestados mostraron estar en general "ni de acuerdo, ni en desacuerdo". El menor valor promedio fue para el indicador 19, el cual refiere a la infraestructura, cuya categoría más repetitiva (moda) fue 1 "en total desacuerdo". Asimismo, el $50 \%$ de los individuos está por encima del valor de 2 y el restante $50 \%$ se sitúa por debajo de este valor (mediana).
La Tabla 5 detalla los estadísticos para la tercera variable.

A continuación, la Figura 3 muestra el promedio obtenido para cada indicador.

Para la variable "Gobernabilidad", los individuos mostraron estar en general "ni de acuerdo, ni en desacuerdo". El menor valor promedio fue para el indicador 30 , el cual refiere a mecanismos y procedimientos institucionalizados. Su categoría más

\section{Variable: Gobierno y gestión}

27. La institucion cuenta con recursos para la.

26. La institucion cuenta con planes de mejoras.

25. Se han tomado en cuenta los diagnósticos y

24. Se han tomado en cuenta las recomendaciones.

23. La institución es capaz de formular.

22. Los sistemas administrativos (recursos.

21. Los sistemas informáticos facilitan el acceso a

20. El equipamiento permite un desarrollo

19. La infraestructura permite un desarrollo.

18. El personal administrativo para las necesidades

17. El personal técnico para las necesidades de

16. Existen resultados correctivos concretos como

15. La comunidad universitaria tiene participacion

14. La organización del gobierno universitario se.

13. La estructura organizacional universitaria se.

12. Existe un conocimiento difundido y entendido.

11. Existen mecanismos para captar la opinión de.

10. Existe informacion disponible para la toma de.

9. La comunidad universitaria tiene participacion.

8. Los procesos decisorios para la adopción de.

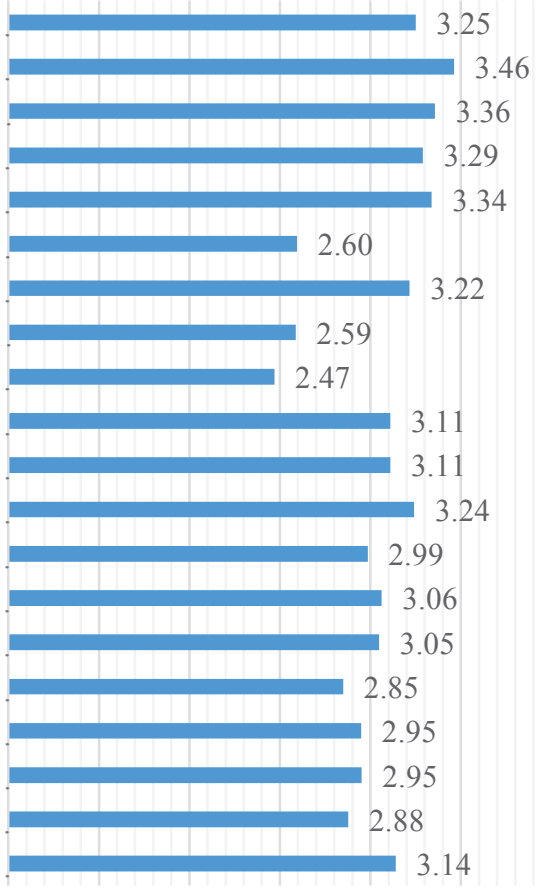

$\begin{array}{lllllllll}1.00 & 1.50 & 2.00 & 2.50 & 3.00 & 3.50 & 4.00 & 4.50 & 5.00\end{array}$

Figura 2. Media de la percepción por indicador.

Fuente: Elaboración propia.

Tabla 5. Percepción sobre los indicadores. Variable: Gobernabilidad.

\begin{tabular}{|c|c|c|c|c|c|}
\hline \multicolumn{2}{|r|}{ Indicador } & \multirow{2}{*}{\begin{tabular}{|c|} 
Media \\
2,75 \\
\end{tabular}} & \multirow{2}{*}{\begin{tabular}{|c|}
$\begin{array}{c}\text { Desviación } \\
\text { estándar }\end{array}$ \\
1,27 \\
\end{tabular}} & \multirow{2}{*}{\begin{tabular}{|c} 
Mediana \\
3
\end{tabular}} & \multirow{2}{*}{\begin{tabular}{|c|} 
Moda \\
4
\end{tabular}} \\
\hline 28 & $\begin{array}{l}\text { Los órganos de gobierno tienen la capacidad de tomar decisiones para la totalidad de la } \\
\text { comunidad universitaria. }\end{array}$ & & & & \\
\hline 29 & $\begin{array}{l}\text { Existe reconocimiento de la forma de gobierno de la institución por parte de la comuni- } \\
\text { dad universitaria. }\end{array}$ & 2,75 & 1,30 & 3 & 4 \\
\hline 30 & $\begin{array}{l}\text { Los mecanismos y procedimientos institucionalizados destinados a facilitar el flujo de } \\
\text { información oportuna a las instancias que corresponda son efectivos. }\end{array}$ & 2,69 & 1,26 & 3 & 4 \\
\hline 31 & Los mecanismos de coordinación entre los distintos órganos de gobierno son efectivos. & 2,70 & 1,29 & 3 & 1 \\
\hline 32 & Las autoridades universitarias tienen capacidad gerencial. & 2,80 & 1,38 & 3 & 1 \\
\hline 33 & $\begin{array}{l}\text { La institución cuenta con criterios respecto a la selección, contratación, perfecciona- } \\
\text { miento y evaluación del personal directivo. }\end{array}$ & 2,89 & 1,33 & 3 & 2 \\
\hline
\end{tabular}

Fuente: Elaboración propia. 


\section{Variable: Gobernabilidad}

33. La institucion cuenta con criterios respecto a la

32. Las autoridades universitarias tienen capacidad.

31. Los mecanismos de coordinación entre los.

30. Los mecanismos y procedimientos

29. Existe reconocimiento a la forma de gobierno

28. Los órganos de gobierno tienen la capacidad de

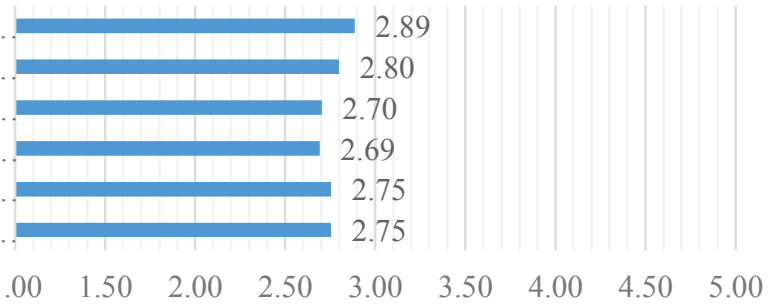

Figura 3. Media de la percepción por indicador.

Fuente: Elaboración propia.

Tabla 6. Percepción sobre los indicadores. Variable: Gestión económico-financiera.

\begin{tabular}{|c|c|c|c|c|c|}
\hline \multicolumn{2}{|r|}{ Indicador } & \multirow{2}{*}{$\begin{array}{c}\text { Media } \\
3,49 \\
\end{array}$} & \multirow{2}{*}{$\begin{array}{c}\begin{array}{c}\text { Desviación } \\
\text { estándar }\end{array} \\
1,12 \\
\end{array}$} & \multirow{2}{*}{$\begin{array}{c}\text { Mediana } \\
4 \\
\end{array}$} & \multirow{2}{*}{$\begin{array}{c}\text { Moda } \\
4 \\
\end{array}$} \\
\hline 34 & Existen normas internas para la elaboración del presupuesto. & & & & \\
\hline 35 & Conozco el presupuesto de la institución. & 2,84 & 1,41 & 3 & 1 \\
\hline 36 & Tengo participación en la elaboración del presupuesto de la institución. & 2,45 & 1,33 & 2 & 1 \\
\hline 37 & Conozco las fuentes de financiamiento del presupuesto de la institución. & 2,82 & 1,39 & 3 & 1 \\
\hline 38 & $\begin{array}{l}\text { El presupuesto es suficiente y equilibrado para atender el conjunto de las activi- } \\
\text { dades académicas. }\end{array}$ & 2,70 & 1,30 & 3 & 4 \\
\hline 39 & $\begin{array}{l}\text { Conozco el procedimiento que se debe llevar a cabo para la asignación de recur- } \\
\text { sos a la institución. }\end{array}$ & 2,76 & 1,35 & 3 & 1 \\
\hline 40 & $\begin{array}{l}\text { Conozco el procedimiento que se debe llevar a cabo para la asignación de recur- } \\
\text { sos por departamento o unidad académica. }\end{array}$ & 2,79 & 1,38 & 3 & 1 \\
\hline 41 & La ejecución presupuestaria se adecúa a los objetivos del proyecto institucional. & 2,89 & 1,27 & 3 & 3 \\
\hline 42 & $\begin{array}{l}\text { Conozco los recursos adicionales que genera la universidad al presupuesto } \\
\text { corriente. }\end{array}$ & 2,78 & 1,35 & 3 & 4 \\
\hline 43 & $\begin{array}{l}\text { Los ámbitos en que se producen los recursos adicionales habitualmente son } \\
\text { vinculación tecnológica, carreras, programas, entre otros. }\end{array}$ & 3,15 & 1,22 & 3 & 4 \\
\hline 44 & Considero que resultan adecuados los mecanismos de gestión de dichos recursos. & 3,11 & 1,12 & 3 & 3 \\
\hline
\end{tabular}

Fuente: Elaboración propia.

repetida (moda) fue 4 con "en parte de acuerdo". Además, el $50 \%$ de los individuos está por encima del valor de 3 y el restante $50 \%$ se sitúa por debajo de este valor (mediana).

En la Tabla 6 se exponen los resultados para la cuarta variable.

Luego, en la Figura 4 se representan los promedios para cada indicador.

Los indicadores de la variable "Gestión económico-financiera" evidenciaron que los individuos mostraron estar en general "ni de acuerdo, ni en desacuerdo". El menor valor promedio fue para el indicador 36 , el cual estaba relacionado con la elaboración del presupuesto institucional. La categoría que más se repitió (moda) fue 1 con "en total desacuerdo". Además, el $50 \%$ de los individuos está por encima del valor de 2 y el restante $50 \%$ se sitúa por debajo de este valor (mediana).
Por consiguiente, en la Tabla 7 se muestran los resultados estadísticos por cada variable.

La Figura 5 muestra los valores medios observados para cada variable.

En promedio, los participantes mostraron estar "ni de acuerdo, ni en desacuerdo" sobre la gestión universitaria. En el menor valor promedial se halla la variable "Gobernabilidad", la cual muestra una menor aceptación de sus afirmaciones por la comunidad académica. Por otro lado, la variable "Misión y proyecto institucional" posee mayor aceptación.

\section{DISCUSIÓN}

Son escasos los instrumentos diseñados para evaluar, medir y caracterizar una determinada gestión universitaria. Una de las razones expuestas que Cejas y Robaina (2012) detectaron fue la complejidad de sus procesos, en los que distintas perspectivas solo se enfocan en las funciones universitarias, 
Variable: Gestión económico-financiera

44. Considero que resultan adecuados los.

43. Los ámbitos en que se producen los recursos..

42. Conozco de los recursos adicionales que.

41. La ejecución presupuestaria se adecúa a los. 40. Conozco el procedimiento que se debe llevar a..

39. Conozco el procedimiento que se debe llevar a..

38. El presupuesto es suficiente y equilibrado para..

37. Conozco las fuentes de financiamiento del

36. Tengo participacion en la elaboracion del

35. Conozco el presupuesto de la institución.

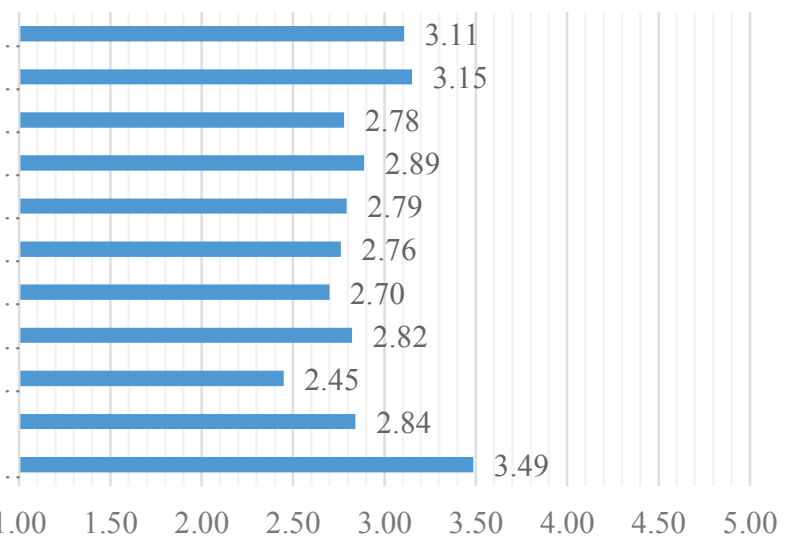

Figura 4. Media de la percepción por indicador.

Fuente: Elaboración propia.

Tabla 7. Percepción sobre la gestión universitaria.

\begin{tabular}{|c|l|c|c|c|}
\hline $\mathbf{N .}^{\circ}$ & \multicolumn{1}{|c|}{ Variable } & Media & Mediana & Moda \\
\hline 1 & Misión y proyecto institucional & 3,74 & 4 & 4 \\
\hline 2 & Gobierno y gestión & 3,04 & 3 & 4 \\
\hline 3 & Gobernabilidad & 2,76 & 3 & 4 \\
\hline 4 & Gestión económico-financiera & 2,89 & 3 & 1 \\
\hline & Gestión universitaria & 3,11 & \multicolumn{2}{|c}{} \\
\cline { 1 - 3 } & &
\end{tabular}

Fuente: Elaboración propia.

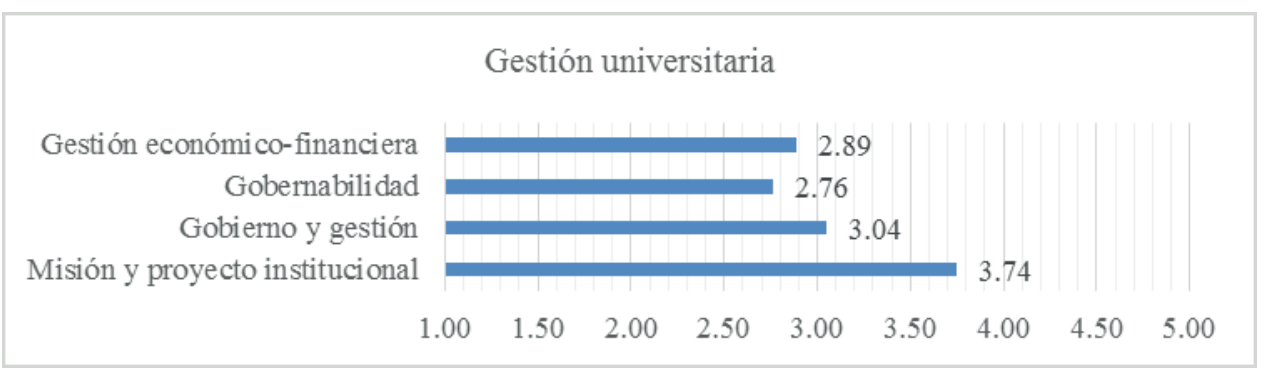

Figura 5. Media de la percepción por variable

Fuente: Elaboración propia.

excluyendo a quienes ejercen el poder; es decir, al poder ejecutivo de la institución (CONEAU, 1998). Asimismo, la gestión universitaria es una práctica transversal, no puede ser encajada en un solo nivel técnico o académico; sino que debe ampliar su observación hacia el gobierno universitario, como señalan Sánchez y López (2013), hacia la práctica de su gestión y el cuestionamiento de las competencias del gestor universitario.
Por tanto, no existen estudios que antecedan a esta investigación, en la que partimos del grupo de variables "Misión y proyecto institucional", "Gobierno y gestión", "Gobernabilidad" y "Gestión económico-financiera", las cuales han sido adaptadas a manera de cuestionario y con escalamiento ordinal. Además, se creó un material que es efectivo y eficaz para establecer propiedades importantes de la misma. El instrumento "Dimensión II. Misión y proyec- 
to institucional. Gobierno y gestión" presenta alta consistencia interna, con un valor alfa de Cronbach de 0,966968 .

\section{Primera variable: Misión y proyecto institucional}

La misión y los objetivos institucionales están definidos en los documentos oficiales de la Universidad Laica Eloy Alfaro de Manabí, como el estatuto, el modelo educativo, el Plan Institucional de Investigación, el Reglamento Orgánico de Gestión Organizacional por Procesos, entre otros. Además, los principales funcionarios los mencionan en actos oficiales, como en la coyuntura del Plan Estratégico de Desarrollo Institucional (PEDI) (ULEAM, 2016b), y son conocidos y aceptados "en parte" por la comunidad universitaria, la cual está integrada por autoridades, personal docente, administrativo y de servicios, y estudiantes (indicadores del 1 al 7 ).

\section{Segunda variable: Gobierno y gestión}

Los procesos decisorios gozan de autonomía institucional, existe información y los mecanismos adecuados para la captura de opiniones. Asimismo, son efectuados mediante el programa informático "Aula virtual", en el que participa la comunidad universitaria (indicadores del 8 al 11).

La actual estructura organizacional está contemplada en el Reglamento Orgánico de Gestión Organizacional por Procesos de esta forma: procesos gobernantes (dirección institucional y estratégica); procesos agregadores de valor (admisión y nivelación, pregrado y posgrado, educación continua, relaciones y cooperación internacional, evaluación interna, planeamiento académico, información bibliográfica y servicios educativos, investigación, vinculación, publicación, y desarrollo cultural); procesos habilitantes de asesoría (jurídica, auditoría interna, organización y métodos, relaciones públicas); procesos habilitantes de apoyo (administrativo, financiero, talento humano, informática, servicios, información académica e institucional).

El presente gobierno universitario o cogobierno es ejercido por el Órgano Colegiado Superior (OCS), el rector, los vicerrectores, las Juntas de Facultad, los Consejos de Facultad, los coordinadores de carrera y directores de Departamentos Centrales de Coordinación. Ambos estamentos son conocidos y se ajustan a los reglamentos y políticas aprobadas; aunque se han observado resultados desde la aplicación de los sistemas de control, la comunidad universitaria no está involucrada en la revisión de la norma que los rige (indicadores del 12 al 16).
Si bien el personal técnico y administrativo es suficiente, la infraestructura y el equipamiento no facilitan las funciones de la academia, lo cual puede ser el resultado del terremoto que se experimentó hace 3 años en la ciudad, en el que colapsaron varios edificios. A pesar de que los sistemas informáticos suministran información, los sistemas administrativos (recursos humanos, adquisiciones y contrataciones, pagos, tesorería o finanzas) no muestran efectividad (indicadores del 17 al 22).

La institución efectúa análisis con el fin de generar políticas, así como toma en cuenta los resultados de evaluaciones previas para la construcción del PEDI, la ejecución de los procesos de autoevaluación institucional, la Evaluación Integral de Desempeño del Personal Académico y la elaboración de planes de mejora con los recursos que dispone (indicadores del 23 al 27).

\section{Tercera variable: Gobernabilidad}

El personal académico que gobierna la universidad tiene capacidad para la toma de decisiones y su régimen es reconocido por la comunidad universitaria (indicadores del 28 y 29 ).

Los mecanismos y procedimientos institucionalizados facilitan el flujo de información; sin embargo, los encuestados indicaron estar "en total desacuerdo" con la efectividad de las principales formas con que la institución coordina el trabajo entre órganos de cogobierno, lo que podría ser la causante de percepción de la incapacidad gerencial de las autoridades (indicadores del 30 al 32 ).

Aunque la universidad disponga del Reglamento Reformatorio y Sustitutivo de Concurso Público de Méritos y Oposición para docentes titulares, se observa "en parte en desacuerdo" con que la institución cuente con criterios de selección, contratación, perfeccionamiento y evaluación del personal directivo (indicador 33).

\section{Cuarta variable: Gestión económico-financiera}

La elaboración del presupuesto esta normado, pero no es socializado hacia la comunidad universitaria, quien no participa en el mismo y desconoce las fuentes del financiamiento (indicadores del 34 al 37).

El presupuesto es suficiente y equilibrado y su ejecución se adecúa a los objetivos institucionales, pero se desconoce el procedimiento que se debe llevar a cabo para la asignación de recursos, ya sea a la institución, departamento o unidad académica (indicadores del 38 al 41). 
Los recursos adicionales que se generan son conocidos y provienen de la vinculación tecnológica, carreras, programas, entre otros, cuyos mecanismos de gestión son adecuados (indicadores del 42 al 44).

\section{CONCLUSIONES}

1. Los estadísticos "mediana" y "moda" proporcionaron a la investigación un nivel correcto de afirmaciones y la encaminó a la toma de decisiones y acciones correctivas.

2. La aplicación del instrumento no solo logró caracterizar el modelo de gestión universitaria de la ULEAM; sino, además, le dio mayor extensión y fortaleza. Si bien, dentro de los subsistemas que la conforman, se puede incluir variables como "Misión y proyecto institucional" o "Gestión económico-financiera", se están obviando elementos como "Gobierno y gestión" y "Gobernabilidad", esenciales por su función analítica sobre el individuo como "juez y parte".

3. El estudio expuso la escasa participación de la comunidad universitaria en los procesos, donde se pudo contar con parte de su juicio. Por tanto, su protagonismo es frágil, pues emite su criterio con imparcialidad. Además, se evidencia que la causa de su indiferencia es producto del desconocimiento (normativa institucional, presupuesto, etc.), así como también lo es el poco compromiso para con la IES (sistemas administrativos, mecanismos de coordinación, capacidad gerencial, concursos de méritos y oposición, etc.).

\section{REFERENCIAS BIBLIOGRÁFICAS}

[1] Álvarez, D., Cejas, J., Robaina, D. y Alonso A. (2013). Modelo de gestión universitaria para las áreas de resultados clave del Instituto Superior politécnico José Antonio Echeverría. Gestión Universitaria, 5(3). Recuperado de http://www. gestuniv.com.ar/gu_15/v5n3a1.htm

[2] Arias, F. (2012). El proyecto de investigación. Introducción a la metodología científica. Caracas, Venezuela: Episteme.

[3] Carbajal, P. (2010). El conflicto en las relaciones interpersonales dentro del colectivo escolar. (Tesis de licenciatura). Universidad Pedagógica Nacional, México D. F.

[4] Cejas, J. y Robaina, D. (2012). Aproximación al estado y tendencias de la Gestión Universitaria en América Latina. Gestión Universitaria, 5(1), s. n. Recuperado de http://www.gestuniv.com. ar/gu_13/v5n1a2.htm

[5] Comisión Nacional de Evaluación y Acreditación Universitaria (1998). Lineamientos para la Evaluación Institucional. Recuperado de http:// www.coneau.gob.ar/archivos/publicaciones/ criteriosymetod/lineamientos.pdf

[6] Comisión Nacional de Evaluación y Acreditación Universitaria (2016). Evaluación institucional. Criterios y procedimientos para la evaluación externa. Recuperado de http://www.coneau. gob.ar/archivos/EvaluacionInstitucional_int_ baja.pdfGarbanzo, G. y Romero, F. (2013). Desafíos y problemáticas de la gestión universitaria en el sistema de la educación superior pública, aspectos a considerar en la región centroamericana. Gestäo Universitária na América Latina, 6(2), 231-258.

[7] Grisales, J. y Monroy, I. (2011). Evaluación del clima organizacional en la Universidad Tecnológica de Pereira y propuestas de intervención para mejorarlo. (Tesis de maestría). Universidad Tecnológica de Pereira, Pereira.

[8] Hernández, R., Fernández, C. y Baptista, P. (2014). Metodología de la investigación. México D.F., México: McGraw-Hill.

[9] Martínez, R. (2000). Evaluación de la gestión universitaria. Informe preparado para la Comisión Nacional de Evaluación y Acreditación Universitaria. Recuperado de https://es.scribd. com/document/360097127/Martinez-NogueiraEvaluacion-de-la-Gestion-Universitaria-pdf

[10] Mujica, M. y Pérez, I. (2009). Construcción de un indicador de gestión fundamentado en el clima organizacional. Revista Venezolana de Gerencia. 14(47), 392-410.

[11] Orlandoni, G. (2010). Escalas de medición en Estadística. Telos. Revista de Estudios Interdisciplinarios en Ciencias Sociales, 12(2), 243-247.

[12] Salazar, C. y Castillo, S. del (2018). Fundamentos básicos de estadística. Quito, Ecuador: s. e.

[13] Sánchez M. y López J. (2013). Buenas prácticas de gobierno y gestión en la universidad. Teoría de la Educación. Revista Interuniversitaria, 25(1), 125-148.

[14] Universidad Laica Eloy Alfaro de Manabí (2014). Estatuto. Recuperado de http://www. 
uleam.edu.ec/wp-content/uploads/2016/11/ ESTATUTO\%20ULEAM(1).pdf

[15] Universidad Laica Eloy Alfaro de Manabí (2014). Reglamento Orgánico de Gestión Organizacional por Procesos. Recuperado de http://www.uleam.edu.ec/wp-content/ u ploads / 2016 / 04 / Reglamen to \% 20 Organico\%20por\%20Procesos1.pdf

[16] Universidad Laica Eloy Alfaro de Manabí (2016). Modelo Educativo. Recuperado de http://www. uleam.edu.ec/wp-content/uploads/2017/01/ ULEAM041-\%20Modelo\%20Educativo\%20 Uleam.pdf.

[17] Universidad Laica Eloy Alfaro de Manabí (2016). Plan Estratégico de Desarrollo Institucional. Recuperado de http://www.uleam.edu.ec/wpcontent/uploads/2018/05/PEDI-2016.-2020. ULEAM_.VERIFICADO2018.1.pdf.

[18] Universidad Laica Eloy Alfaro de Manabí (2016). Plan Institucional de Investigación
2016-2020. Recuperado de http://www. uleam.edu.ec/wp-content/uploads/2017/01/ PLAN\%20INSTITUCIONAL \% 20DE\% 20 INVESTIGACION\%202016-2020.pdf.

[19] Universidad Laica Eloy Alfaro de Manabí (2016). Reglamento de Evaluación Integral del Desempeño del Personal Académico. Recuperado de http://www.uleam.edu.ec/wpcontent/uploads/2017/01/ULEAM015-\%20 REGLAMENTO - DE - EVALUACION INTEGRAL-DEL-DESEMPENO-DELPERSONAL-ACADEMICO.pdf.Universidad Laica Eloy Alfaro de Manabí (2016). Reglamento Reformatorio y Sustitutivo de Concurso Público de Méritos y Oposición para Docentes Titulares. Recuperado de http://www.uleam.edu.ec/ wp-content/uploads/2018/07/ULEAM019RE G LAMENTO - RE F O RMATORIO Y-SUSTITUTIVO-DE - CONCURSOM\%C3\%89RITO-Y-OPOSICI\%C3\%93N-CONLA-DEROGACI\%C3\%93N.pdf. 


\section{Characterization of university management within the Universidad Laica Eloy Alfaro De Manabi (ULEAM), Ecuador}

\begin{abstract}
This article focuses on one of the most significant problems of higher education in Ecuador: university management. Its definition is the only element designed with an academic perspective, since administrative actions have not been included and there is a lack of information regarding its components, without which no examination or characterization is possible. As a result, this study analyzes and describes management at the Universidad Laica Eloy Alfaro de Manabí (ULEAM) via the implementation of "Dimensión II. Misión y proyecto institucional. Gobierno y gestión" [Dimension II. Institutional mission and project. Government and management], which integrates the tool used by the Comisión Nacional de Evaluación y Acreditación Universitaria [National Commission for University Evaluation and Accreditation] (CONEAU) of Argentina in their evaluation processes. This tool has been adapted to the Likert scale survey in order to collect data gathered from faculty and non-faculty members. Finally, the results showed a serious weakness in government, management and governability factors.
\end{abstract}

Keywords: Organization; university management; higher education; governabilityt.

\section{INTRODUCTION}

An organization is a group of people with an established structure that, together with its characteristics, meets a number of set common objectives (Carbajal, 2010). Accordingly, universities are also organizations with structures, responsibilities and demands, in which the categorical action of their members and authority guidance are relevant (Grisales \& Monroy, 2011). If an organization maintains a reciprocal relationship between people who coordinate resources, processes, and a human component using a dynamic system, then an adequate university management will promote academic processes efficiency, ensure quality, and will lead to the awarding of university accreditation. Consequently, it will be possible to align the performance of each person and unit of its structure towards the policy and institutional strategies, for the purpose of being able to base these components on clear norms and procedures that prevent repetitive or ambiguous functions, and present sufficient resources and relevant competences

The process of managing the activities of an organization with a high degree of responsibility and other related characteristics is referred to as institutional management; however, when the institution subjected to this action is a university, it is known as university management. Management is a set of tasks performed in order to obtain a product of an administrative nature, hence this term is more often used in scheduled and planned activities. Its concept and application widen with the inclusion of the term leadership, in addition to granting it a greater scope and not limiting it to the compliance of activities related to the four basic management processes (planning, organizing, leading and controlling). One of the most important roles of university management is the management of the academy, to which science, administration and culture must be added to contribute to the fulfillment of its role (Cejas \& Robaina, 2012). On this basis, a re-

1 Master in Educational Management from the Universidad Estatal del Sur de Manabí (Ecuador), she studied Industrial Engineering at the Universidad Laica Eloy Alfaro de Manabí (Ecuador) and is Professor at the same institution. Manabí-Ecuador.

E-mail: jahaira.quijije@uleam.edu.ec

2 Master in Business and Institutional Communication Management from the Universidad de las Américas (Ecuador), has a degree in Social Communication with a specialization in Organizational Communication and Public Relations from the Universidad Laica Eloy Alfaro de Manabí (Ecuador). She is a teacher at the school Unidad Educativa del Milenio Dra. Guadalupe Larriva (Ecuador). Manabí-Ecuador. E-mail: kerly.quijije@educacion.gob.ec 
lationship between administration and management is essential, as it will contribute with the enhancement of the latter.

In 1998, the Comisión Nacional de Evaluación y Acreditación Universitaria [National Commission for University Evaluation and Accreditation] (CONEAU) of Argentina took a step forward, as it characterized university management from the perspective of the time, that is, understood from the structuring of functions and the management of resources available for the purpose. It is important to recall that university should not only be understood as an organization, but as an institution of broad and dynamic scope, in which its component elements are to be considered from a human perspective as a result of formal education (Mujica \& Pérez, 2009). In this way, the creation of university institutions has evolved to conceptualize the university and, therefore, its management, bearing in mind its history (Cejas \& Robaina, 2012; Álvarez, Cejas, Robaina \& Alonso, 2013). At present, the modern university is obliged to constantly review its management model with the aim of redirecting its activities so that they are better suited to new educational demands (Garbanzo \& Romero, 2013).

This study is part of an investigation that relates two subjects connected with higher education: university management, defined as the process of planning, organizing, leading and controlling, in other words, applying the pillars of management with a high degree of diligence, responsibility and efficiency; and organizational climate, known as the employees' perception of their working environment, which has an impact on their productivity. Also, this topic provides greater understanding of the difference between the definitions of administration and management, as it aims at presenting the reader, and the university community in particular, the state of institutional management at the ULEAM in Ecuador. Also, the subject provides a better understanding on the difference between administration and management, as it aims at presenting the reader, and university community in particular, information about the state of institutional management at the ULEAM in Ecuador.
The study relates to the theoretical approach, where the ULEAM university management components are analyzed and characterized. Likewise, this contributes to its conceptualization as an institution, directs its administrative and educational system, promotes user satisfaction, addresses the needs of the working environment, and sets a precedent for the incorporation of the organizational approach into its processes. One of the most significant limitations, however, was encountered during the data collection stage, in which a certain degree of fear of answering the survey questions was observed among the respondents, specifically regarding the questions regarding government and governability.

\section{METHODOLOGY}

This is an exploratory study, as management at the university is instrumental and poses itself as an object of study. It is also descriptive, because data on the variables is collected in order to establish characteristics and enable the discrimination of relevant factors. Similarly, it is non-experimental because variables are not manipulated (Hernández, Fernández \& Baptista, 2014).

Similarly, the population of 928 individuals is comprised of faculty and non-faculty members employed at ULEAM. Likewise, the sample size was determined using a finite population equation, with $95 \%$ reliability and $5 \%$ margin of error; a probabilistic, stratified sampling technique is used (Hernández, Fernández \& Baptista, 2014). A total of 272 valid questionnaire surveys were thereby gathered, as shown in Table 1.

The technique used for data collection was the written survey which is, as defined by Arias (2012), a questionnaire with closed-ended questions. The "Dimension II. Institutional mission and project. Government and management." instrument was used, which is part of the set of dimensions that comprise the instrument Evaluación institucional: criterios y procedimientos para la evaluación externa [Institutional evaluation: criteria and procedures for external evaluation], which results from the adjustment made to Lineamientos para la evaluación

Table 1. Sample data.

\begin{tabular}{|l|c|c|}
\hline \multicolumn{1}{|c|}{ Strata } & No. of respondents & Percentage \\
\hline Faculty members: teaching staff & 215 & $79 \%$ \\
\hline Non-faculty members: administrative and service staff & 57 & $21 \%$ \\
\hline Total & 272 & $100 \%$ \\
\hline
\end{tabular}

Source: Prepared by the authors. 
institucional [Guidelines for institutional evaluation]. Originally, it consisted only of three variables, to which governability was added, as proposed in Evaluación de la gestión universitaria [Evaluation of university management] for the purpose of procuring more information about the governing bodies. The final instrument is detailed in Table 2.

A Likert scale, being a current and popular instrument in studies that measure perceptions, was adapted to the questionnaire, with categories ranging from 1 to 5 , where 1 means "Strongly disagree" and 5 means "Completely agree".

The following was performed regarding construct validity:

- A specialized literature review from 2009 to the present date.

- A comparison between administration and management based on eight authors from 2000, and from 2009 to the present day.

- An analysis of four criteria with 22 dimensions of socialization.

- A review of eight measurement models, with three variables and over 465 indicators, including type of business management, educational management and a European model.

- A review of data obtained from previous meetings of the Coloquios Internacionales de Gestión Universitaria [International Symposium on University Management] (CIGU) starting from the year 2000. This event is organized in association with the Universidad Federal de Santa Catarina (UFSC), the Instituto de Investigaciones y Estudios en Administración Universitaria (INPEAU), the Universidad Nacional de Mar del Plata (UNMDP) and the Asociación de Especialistas en Gestión de la Educación Superior (AEGES) in Argentina.

- Studies on management practice and manager.

- A review of eight criteria regarding university management competences.

- An adaptation of the original material using specialized literature.

Cronbach's alpha was the statistic used to measure internal consistency. In addition, the statistical software SPSS was used for data processing.

\section{RESULTS}

In attitudinal studies, the mean can be affected by atypical values that destabilize it; therefore, the analysis of results heavily relies on the median and mode, as Orlandoni (2010) recommends for measurement scales with ordinal data, due to these being highly representative of the sample (Salazar \& Castillo, 2017).

Table 2. Instrument used for the characterization of university management

\begin{tabular}{|c|l|l|c|}
\hline \multicolumn{1}{|c|}{ Model } & \multicolumn{1}{|c|}{ Author } & \multicolumn{1}{c|}{ Variables } & Indicators \\
\hline $\begin{array}{l}\text { Dimension II. Institutional mission and pro- } \\
\text { ject. Government and management (2016). }\end{array}$ & $\begin{array}{l}\text { Comisión Nacional de Evaluación y } \\
\text { Acreditación Universitaria (CONEAU) }\end{array}$ & $\begin{array}{l}\text { 1. Institutional mission and project } \\
\text { 2. Government and Management } \\
\text { 3. Goovernability } \\
\text { 4.Economic-financial management }\end{array}$ & 44 \\
\hline
\end{tabular}

Source: Prepared by the authors, based on information obtained from CONEAU (1998), CONEAU (2016) and Martínez (2000).

Table 3. Perception Indicators. Variable: Mission and institutional project.

\begin{tabular}{|c|c|c|c|c|c|}
\hline \multicolumn{2}{|r|}{ Indicator } & \multirow{2}{*}{$\begin{array}{c}\text { Mean } \\
3.81\end{array}$} & \multirow{2}{*}{$\begin{array}{c}\begin{array}{c}\text { Standard } \\
\text { deviation }\end{array} \\
1.32\end{array}$} & \multirow{2}{*}{$\frac{\text { Median }}{4}$} & \multirow{2}{*}{$\begin{array}{c}\text { Mode } \\
5\end{array}$} \\
\hline 1 & The institutional mission is clearly defined in official documents. & & & & \\
\hline 2 & The institutional objectives are clearly defined in official documents. & 3.81 & 1.27 & 4 & 5 \\
\hline 3 & $\begin{array}{l}\text { The institutional development plan and/or strategic plan is connected with the mission } \\
\text { and institutional objectives. }\end{array}$ & 3.75 & 1.18 & 4 & 4 \\
\hline 4 & The university community knows and accepts the institutional mission. & 3.74 & 1.14 & 4 & 4 \\
\hline 5 & The university community knows and accepts the institutional objectives. & 3.61 & 1.13 & 4 & 4 \\
\hline 6 & The mission is properly expressed by the main actors and in every academic activity. & 3.77 & 1.08 & 4 & 4 \\
\hline 7 & $\begin{array}{l}\text { The institutional objectives are properly expressed by the main actors and in every } \\
\text { academic activity. }\end{array}$ & 3.73 & 1.12 & 4 & 4 \\
\hline
\end{tabular}

Source: Prepared by the authors. 
Table 3 displays the parameters for the first variable.

Figure 1 shows the mean value obtained for each item.

Respondents indicated they "Partially agree" with the indicators of variable "Institutional mission and project". The lowest mean value corresponded to indicator 5, which refers to institutional objectives. Level 4, "Partially agree", was the category that appeared most often (mode). Also, $50 \%$ of the response is above value 4 , and the remaining $50 \%$ is below this value (median).

Variable: Institutional mission and project

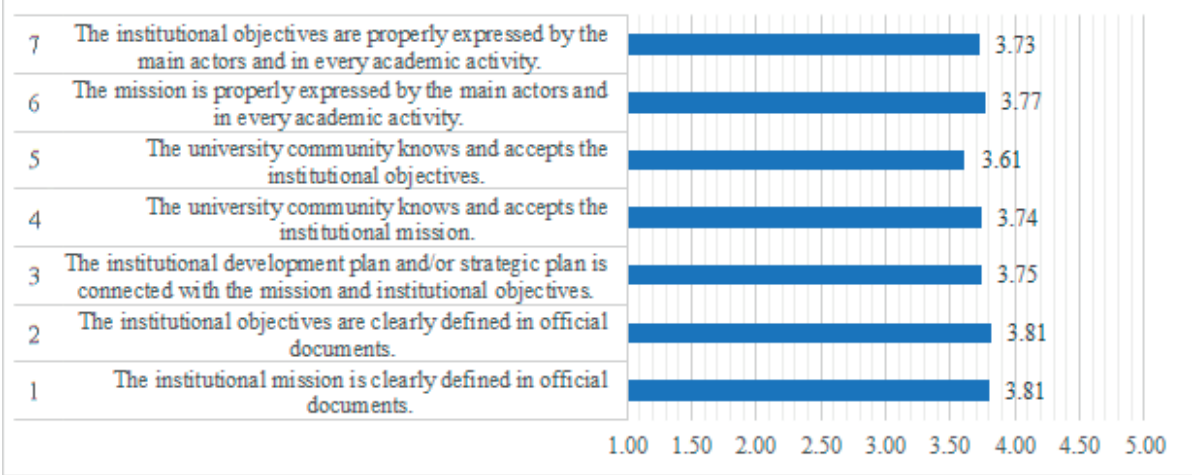

Figure 1. Mean perception per item.

Source: Prepared by the authors.

Table 4. Perception Indicators. Variable: Government and management.

\begin{tabular}{|c|c|c|c|c|c|}
\hline \multicolumn{2}{|c|}{ Indicator } & \multirow{2}{*}{$\begin{array}{c}\text { Mean } \\
3.14\end{array}$} & \multirow{2}{*}{$\begin{array}{c}\begin{array}{c}\text { Standard } \\
\text { deviation }\end{array} \\
1.32 \\
\end{array}$} & \multirow{2}{*}{$\begin{array}{c}\text { Median } \\
3\end{array}$} & \multirow{2}{*}{ Mode } \\
\hline 8 & Decision-making processes for policymaking have academic and institutional autonomy. & & & & \\
\hline 9 & The university community participates in decision-making processes. & 2.88 & 1.35 & 3 & 4 \\
\hline 10 & Information for decision-making is available. & 2.95 & 1.45 & 3 & 4 \\
\hline 11 & $\begin{array}{l}\text { There are mechanisms to collect the opinion of pupils, professors and administrative staff } \\
\text { on pertinent decision-making aspects. }\end{array}$ & 2.95 & 1.34 & 3 & 4 \\
\hline 12 & $\begin{array}{l}\text { There is widespread knowledge and understanding of the current university organizatio- } \\
\text { nal structure. }\end{array}$ & 2.85 & 1.33 & 3 & 4 \\
\hline 13 & The university organizational structure conforms to institutional regulations. & 3.05 & 1.29 & 3 & 4 \\
\hline 14 & The organization of the university government conforms to institutional regulations. & 3.06 & 1.31 & 3 & 4 \\
\hline 15 & The university community participates in the review of institutional regulations. & 2.99 & 1.33 & 3 & 2 \\
\hline 16 & Concrete corrective results follow from the implementation of management control systems. & 3.24 & 1.17 & 3 & 4 \\
\hline 17 & There is sufficient technical staff to address management needs. & 3.11 & 1.17 & 3 & 4 \\
\hline 18 & There is sufficient administrative staff to address management needs. & 3.11 & 1.32 & 3 & 4 \\
\hline 19 & $\begin{array}{l}\text { The infrastructure allows the proper development of teaching, researching and university } \\
\text { extension functions. }\end{array}$ & 2.47 & 1.29 & 2 & 1 \\
\hline 20 & $\begin{array}{l}\text { The equipment allows the proper development of teaching, researching and university } \\
\text { extension functions. }\end{array}$ & 2.59 & 1.29 & 2 & 2 \\
\hline 21 & $\begin{array}{l}\text { Computer systems provide easy access to information for decision-making and manage- } \\
\text { ment control. }\end{array}$ & 3.22 & 1.18 & 3 & 4 \\
\hline 22 & $\begin{array}{l}\text { Administrative systems (human resources, purchasing, acquisitions and hiring, pay- } \\
\text { ments, settlements and expensing, accounting, financial and asset management, etc.) } \\
\text { are effective. }\end{array}$ & 2.60 & 1.25 & 2 & 2 \\
\hline 23 & $\begin{array}{l}\text { The institution is capable of making diagnoses and creating policies within the framework } \\
\text { of strategic planning. }\end{array}$ & 3.34 & 1.10 & 4 & 4 \\
\hline 24 & $\begin{array}{l}\text { Recommendations of previous institutional evaluations were considered in the making of } \\
\text { institutional development plans and/or strategic plan. }\end{array}$ & 3.29 & 1.14 & 3 & 4 \\
\hline 25 & $\begin{array}{l}\text { Diagnoses and recommendations of previous institutional evaluations were considered in } \\
\text { the self-evaluation process. }\end{array}$ & 3.36 & 1.12 & 4 & 4 \\
\hline 26 & The institution has improvement plans for different functions. & 3.46 & 1.18 & 4 & 4 \\
\hline 27 & The institution has resources for the planning of different functions. & 3.25 & 1.17 & 3 & 4 \\
\hline
\end{tabular}

Source: Prepared by the authors. 
Table 4 shows the results obtained for the second variable.

Next, Figure 2 shows the mean value obtained for each variable indicator: Respondents in general indicated they "Neither agree nor disagree" with the indicators of variable "Government and management". The lowest mean value corresponded to indicator 19, which refers to infrastructure, where level 1, "Strongly disagree" was the category that appeared most often (mode). Also, $50 \%$ of the response is above value 2 , and the remaining $50 \%$ is below this value (median).
Table 5 shows the results obtained for the third variable.

Next, Figure 3 shows the mean value for each indicator.

Respondents in general indicated they "Neither agree nor disagree" with the indicators of variable "Governability". The lowest mean value corresponded to indicator 30 , which refers to institutionalized mechanisms and procedures. Level 4, "Partially agree" was the category that appeared most often (mode). Also, $50 \%$ of the response is above value 3 , and the remaining $50 \%$ is below this value (median).

\section{Variable: Government and management}

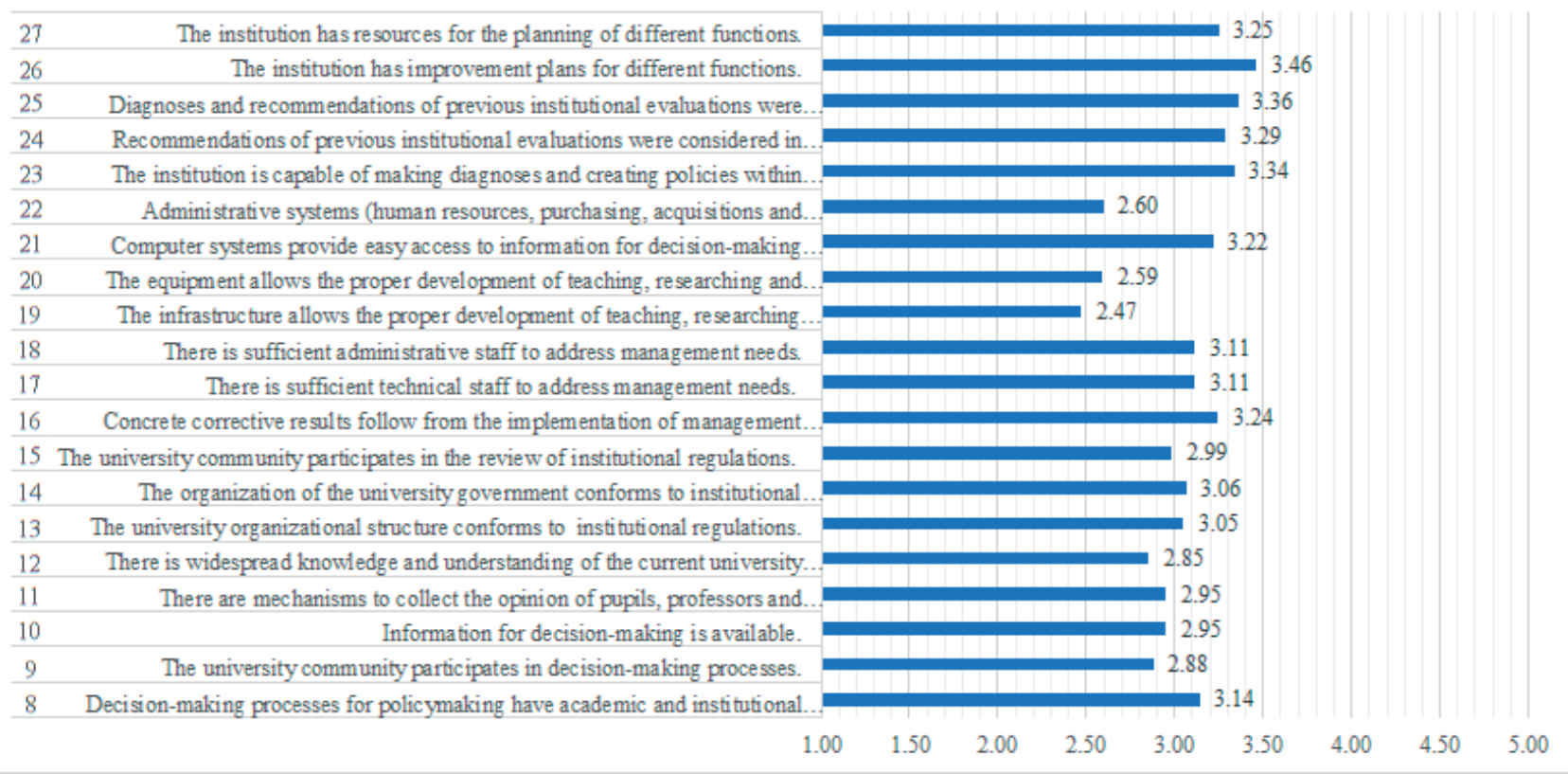

Figure 2. Mean perception per indicator. Source: Prepared by the authors.

Table 5. Perception Indicators. Variable: Governability.

\begin{tabular}{|c|c|c|c|c|c|}
\hline \multicolumn{2}{|r|}{ Indicator } & \multirow{2}{*}{$\frac{\text { Mean }}{2.75}$} & \multirow{2}{*}{$\begin{array}{c}\begin{array}{c}\text { Standard } \\
\text { deviation }\end{array} \\
1.27\end{array}$} & \multirow{2}{*}{$\frac{\text { Median }}{3}$} & \multirow{2}{*}{$\frac{\text { Mode }}{4}$} \\
\hline 28 & $\begin{array}{l}\text { The governing bodies have the power to make decisions for the entire university com- } \\
\text { munity. }\end{array}$ & & & & \\
\hline 29 & The university community recognizes the form of government of the institution. & 2.75 & 1.30 & 3 & 4 \\
\hline 30 & $\begin{array}{l}\text { Institutionalized mechanisms and procedures intended to facilitate the flow of relevant } \\
\text { information to the corresponding instances are effective. }\end{array}$ & 2.69 & 1.26 & 3 & 4 \\
\hline 31 & Coordination mechanisms between the various governing bodies are effective. & 2.70 & 1.29 & 3 & 1 \\
\hline 32 & University authorities have managerial capacity & 2.80 & 1.38 & 3 & 1 \\
\hline 33 & $\begin{array}{l}\text { The institution has established criteria regarding selection, hiring, improvement and } \\
\text { evaluation of executives. }\end{array}$ & 2.89 & 1.33 & 3 & 2 \\
\hline
\end{tabular}

Source: Prepared by the authors. 


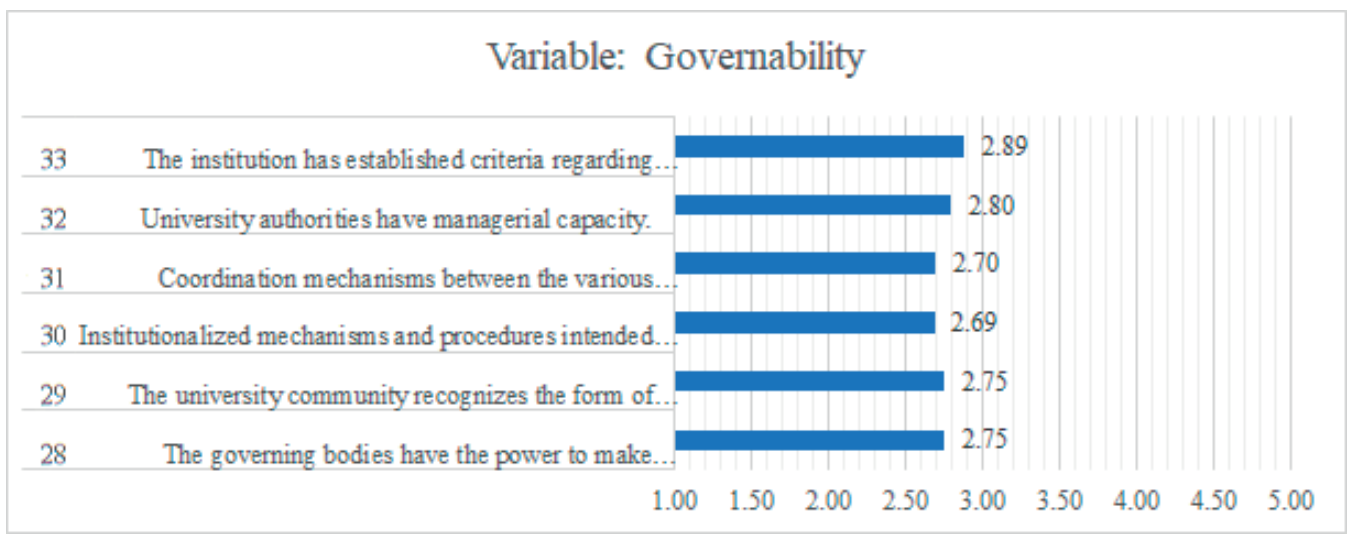

Figure 3. Mean perception per indicator.

Source: Prepared by the author.

Table 6. Perception Indicators. Variable: Economic and financial management.

\begin{tabular}{|c|c|c|c|c|c|}
\hline \multicolumn{2}{|r|}{ Indicator } & \multirow{2}{*}{$\begin{array}{r}\text { Mean } \\
3.49 \\
\end{array}$} & \multirow{2}{*}{$\begin{array}{r}\begin{array}{r}\text { Standard } \\
\text { deviation }\end{array} \\
1.12\end{array}$} & \multirow{2}{*}{$\begin{array}{c}\text { Mediana } \\
4\end{array}$} & \multirow{2}{*}{$\frac{\text { Moda }}{4}$} \\
\hline 34 & There are internal procedures for the drafting of the budget. & & & & \\
\hline 35 & I am familiar with the institution's budget. & 2.84 & 1.41 & 3 & 1 \\
\hline 36 & I am involved in the drafting of the budget. & 2.45 & 1.33 & 2 & 1 \\
\hline 37 & I am familiar with the funding sources of the institution's budget. & 2.82 & 1.39 & 3 & 1 \\
\hline 38 & The budget is balanced and sufficient to cover all the academic activities. & 2.70 & 1.30 & 3 & 4 \\
\hline 39 & $\begin{array}{l}\text { I am familiar with the procedure that must be carried out for resource allocation to } \\
\text { the institution. }\end{array}$ & 2.76 & 1.35 & 3 & 1 \\
\hline 40 & $\begin{array}{l}\text { I am familiar with the procedure that must be carried out for resource allocation } \\
\text { by departments or academic units. }\end{array}$ & 2.79 & 1.38 & 3 & 1 \\
\hline 41 & Budget implementation adjusts to the institutional project objectives. & 2.89 & 1.27 & 3 & 3 \\
\hline 42 & $\begin{array}{l}\text { I am familiar with the additional resources generated by the university for the } \\
\text { recurrent budget. }\end{array}$ & 2.78 & 1.35 & 3 & 4 \\
\hline 43 & $\begin{array}{l}\text { The areas in which additional resources are generated are usually technology } \\
\text { transfer, professional careers, programs, among others. }\end{array}$ & 3.15 & 1.22 & 3 & 4 \\
\hline 44 & I believe that the management mechanisms of said resources are appropriate. & 3.11 & 1.12 & 3 & 3 \\
\hline
\end{tabular}

Source: Prepared by the author.

Table 6 shows the results obtained for the fourth variable.

Figure 4 shows the mean value obtained for each indicator.

Respondents in general indicated they "Neither agree nor disagree" with the indicators of variable "Economic and financial management". The lowest mean value corresponded to indicator 36 , which refers to the drafting of the institutional budget. Level 1, "Completely agree" was the category that appeared most often (mode). Also, $50 \%$ of the response is above value 2 , and the remaining $50 \%$ is below this value (median).

Therefore, Table 7 show the statistical values obtained for each variable.

Figure 5 shows the mean value obtained for each variable.
Respondents in general indicated they "Neither agree nor disagree" with university management. The lowest mean value corresponded to variable "governability", whose statements were less accepted by the university community. Conversely, variable "Institutional mission and project" has a higher degree of acceptance.

\section{DISCUSSION}

There are few instruments designed to evaluate, measure and characterize the university management of a specific institution. One of the reasons provided by Cejas and Robaina (2012) was the complexity of its processes, where different perspectives are only focused on university functions, excluding those in power; in other words, the executive branch of the institution (CONEAU, 1998). Additionally, university management is a cross-cutting practice that cannot be encompassed by only 


\section{Variable: Economic and financial management}

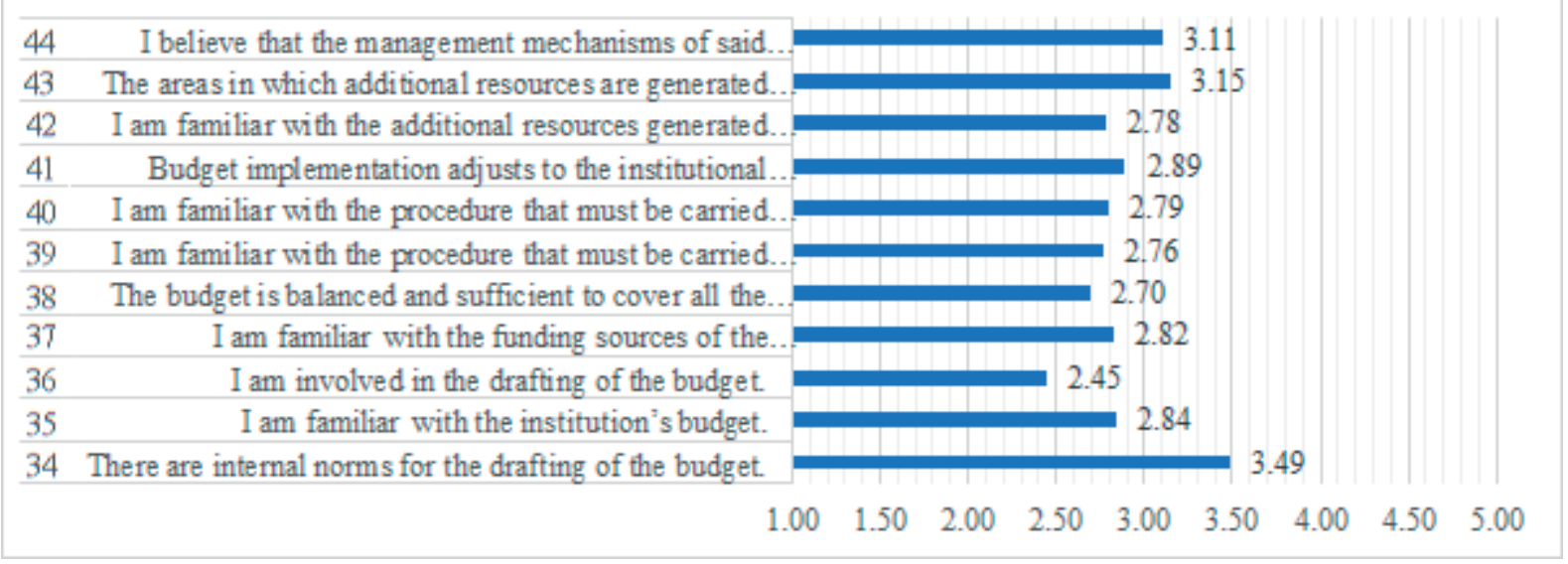

Figure 4. Mean perception per indicator.

Source: Prepared by the author.

Table 7. Perception of university management.

\begin{tabular}{|c|l|c|c|c|}
\hline $\mathbf{N .}^{\circ}$ & \multicolumn{1}{|c|}{ Variable } & Mean & Median & Mode \\
\hline 1 & Institutional mission and project & 3.74 & 4 & 4 \\
\hline 2 & Government and management & 3.04 & 3 & 4 \\
\hline 3 & Governability & 2.76 & 3 & 4 \\
\hline 4 & Economic and financial management & 2.89 & 3 & 1 \\
\hline & University management & 3.11 & \multicolumn{2}{|c}{} \\
\cline { 1 - 3 } & &
\end{tabular}

Source: Prepared by the authors.

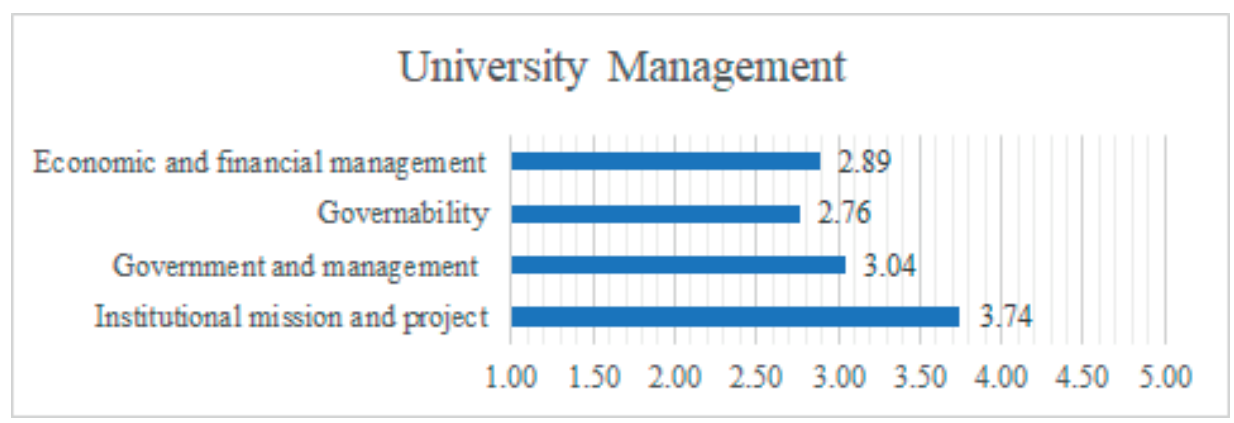

Figure 5. Mean perception per item

Source: Prepared by the authors.

one technical or academic level; rather, its study should be expanded to the entire context of university governability, and to the practice and questioning of university manager competencies, as stated by Sánchez and López (2013).

Therefore, there are no previous studies regarding this research that includes variables "Institutional mission and project", "Government and management", "Governability" and "Economic and financial management", which were adapted as a question- naire with ordinal scale. An effective and efficient tool to determine important properties of the study was created. The instrument "Dimension II. Institutional project and mission". Government and management" has high internal consistency with a Cronbach's alpha coefficient of 0.966968 .

\section{First variable: Institutional mission and project}

Both institutional mission and objectives are defined in official documents of the Universidad Laica Eloy 
Alfaro de Manabí, such as the statute, educational model, the Plan Institucional de Investigación [Institutional Research Plan], the Reglamento Orgánico de Gestión Organizacional por Procesos [Organic Statute Organizational Management by Processes], among others. Furthermore, main university authorities refer to them in official acts such as the Plan Estratégico de Desarrollo Institucional [Strategic Plan for Institutional Development] (PEDI) (ULEAM, 2016b), and they are "partially" accepted by the university community comprised of authorities, faculty, administrative and service staff, and pupils (indicators 1 to 7 ).

\section{Second variable: Government and management}

Decision-making processes have institutional autonomy, information is available and there are mechanisms to collect opinions. In addition, they are conducted through the software program "Aula virtual", which the entire university community uses (indicators 8 to 11).

The current organizational structure is accounted for in the Reglamento Orgánico de Gestión Organizacional por Procesos [Organic Statute Organizational Management by Processes] as follows: governing processes (institutional and strategic direction), value adding processes (admission and levelling, undergraduate studies and postgraduate studies, continuous education, international relations and cooperation, internal evaluation, academic planning, bibliographic information and educational services, research, technology transfer, publication, and cultural development), advice enabling processes (legal advice, internal audit, organization and methods, public relations); support enabling processes (administrative, financial, human talent, information technology, services, academic and institutional information).

The current university government or co-government is exercised by the Órgano Colegiado Superior $^{\mathrm{TN} 1}$ (OCS), chancellor, vice-chancellors, faculty boards, faculty councils, career coordinators ${ }^{\mathrm{TN} 2}$ and heads of Central Coordination Departments. Both, government and management, are known and conform to the approved regulations and policies. Although results have been observed since the implementation of control systems, university community does not participate in the review of the regulations governing them (indicators 12 to 16).

\footnotetext{
TN1: Maximum authority at higher education institutions composed of authorities, and representatives of both professors and students.

TN2: Professor in charge of planning and coordinating the study curriculum, as well as academic, cultural and sport activities.
}

Whereas technical and administrative staff is sufficient, infrastructure and equipment fail to facilitate the functions of the academy, which might be the result of the earthquake experienced in the city three years ago that caused the collapse of several buildings. Although computer systems provide information, administrative systems (human resources, acquisitions and hiring, payments, finance or treasury) fail to prove efficiency (indicator 17 to 22).

The institution performs analyses in order to generate policies; it also considers results obtained from previous evaluations to elaborate the strategic plan for institutional development (PEDI), the execution of institutional self-evaluation processes, the comprehensive evaluation of academic personnel and the elaboration of plans of improvement using the resources available (indicators 23 to 27 ).

\section{Third variable: Governability}

The academic staff governing the university has the capacity to make decisions and its authority is recognized by the university community (indicator 28 to 29 ).

Institutionalized mechanisms and procedures facilitate the flow of information; however, respondents stated they "Strongly disagree" with the effectiveness of the main methods used by the institution to coordinate the work among co-government bodies, which might be the reason due to which there is a perception of managerial inability of authorities (indicators 30 to 32 ).

Although the university has the Reglamento Reformatorio y Sustitutivo de Concurso Público de Méritos y Oposición ${ }^{\mathrm{TN} 3}$ for professors, respondents "partly disagree" with the institution having criteria regarding the selection, hiring, improvement and evaluation of executives (indicator 33 ).

\section{Fourth variable: Economic and financial man- agement}

The drafting of the budget is regulated, however, it is not shared with the university community, who does not take part of this process and is unaware of the source of funding (indicators 34 to 37 ).

The budget is balanced and sufficient and its execution adjusts to the institutional objectives, the procedure that must be carried out for resource allocation to an institution, academic department or academic unit is not common knowledge (indicators 38 to 41).

TN3: Regulatory document that establishes the compulsory procedure for academic staff selection. 
Additional resources generated are known and come from technological transfer, professional careers, programs, among others, whose management mechanisms are appropriate (indicators 42 to 44).

\section{CONCLUSIONS}

1. The "median" and "mode" provided an appropriate level of statements to the study and directed it to decision-making and corrective measures.

2. The implementation of the instrument not only characterized the university management model of ULEAM, but it also provides it with a greater scope and strength. Although variables such as "Institutional mission and project" or "Economic and financial management" may be included within the subsystems that comprise said model, elements such as "Government and management" and "Governability", essential for their analytical function regarding individuals as "judge and jury", are ignored.

3. The study, which included the university community, demonstrated the low level of participation by the university community in university management processes. Therefore, their involvement is minimal, and as such their opinion is impartial. Also, it is evident that such indifference results from the lack of knowledge (regarding institutional regulations, budget, etc.), and from the lack of commitment towards the higher education institution (administrative systems, coordination mechanisms, managerial capacity, competitive examinations, etc.).

\section{REFERENCES}

[1] Álvarez, D., Cejas, J., Robaina, D. \& Alonso A. (2013). Modelo de gestión universitaria para las áreas de resultados clave del Instituto Superior politécnico José Antonio Echeverría. Gestión Universitaria, 5(3). Retrieved from http://www. gestuniv.com.ar/gu_15/v5n3a1.htm

[2] Arias, F. (2012). El proyecto de investigación. Introducción a la metodología científica. Caracas, Venezuela: Episteme.

[3] Carbajal, P. (2010). El conflicto en las relaciones interpersonales dentro del colectivo escolar. (Bachelor Thesis). Universidad Pedagógica Nacional, Mexico D. F.

[4] Cejas, J. \& Robaina, D. (2012). Aproximación al estado y tendencias de la Gestión Universitaria en América Latina. Gestión Universitaria, 5(1), s. n. Retrieved from http://www.gestuniv.com. ar/gu_13/v5n1a2.htm

[5] Comisión Nacional de Evaluación y Acreditación Universitaria (1998). Lineamientos para la Evaluación Institucional. Retrieved from http:// www.coneau.gob.ar/archivos/publicaciones/ criteriosymetod/lineamientos.pdf

[6] Comisión Nacional de Evaluación y Acreditación Universitaria (2016). Evaluación institucional. Criterios y procedimientos para la evaluación externa. Retrieved from http://www.coneau. gob.ar/archivos/EvaluacionInstitucional_int_ baja.pdfGarbanzo, G. y Romero, F. (2013). Desafíos y problemáticas de la gestión universitaria en el sistema de la educación superior pública, aspectos a considerar en la región centroamericana. Gestäo Universitária na América Latina, 6(2), 231-258.

[7] Grisales, J. \& Monroy, I. (2011). Evaluación del clima organizacional en la Universidad Tecnológica de Pereira y propuestas de intervención para mejorarlo. (Tesis de maestría). Universidad Tecnológica de Pereira, Pereira.

[8] Hernández, R., Fernández, C. \& Baptista, P. (2014). Metodología de la investigación. Mexico D.F., Mexico: McGraw-Hill.

[9] Martínez, R. (2000). Evaluación de la gestión universitaria. Informe preparado para la Comisión Nacional de Evaluación y Acreditación Universitaria. Retrieved from https://es.scribd. com/document/360097127/Martinez-NogueiraEvaluacion-de-la-Gestion-Universitaria-pdf

[10] Mujica, M. \& Pérez, I. (2009). Construcción de un indicador de gestión fundamentado en el clima organizacional. Revista Venezolana de Gerencia. 14(47), 392-410.

[11] Orlandoni, G. (2010). Escalas de medición en Estadística. Telos. Revista de Estudios Interdisciplinarios en Ciencias Sociales, 12(2), 243-247.

[12] Salazar, C. \& Castillo, S. del (2018). Fundamentos básicos de estadística. Quito, Ecuador: s. e.

[13] Sánchez M. \& López J. (2013). Buenas prácticas de gobierno y gestión en la universidad. Teoría de la Educación. Revista Interuniversitaria, 25(1), 125-148.

[14] Universidad Laica Eloy Alfaro de Manabí (2014). Estatuto. Retrieved from http://www. 
uleam.edu.ec/wp-content/uploads/2016/11/ ESTATUTO\%20ULEAM(1).pdf

[15] Universidad Laica Eloy Alfaro de Manabí (2014). Reglamento Orgánico de Gestión Organizacional por Procesos. Retrieved from http://www.uleam.edu.ec/wp-content/ uploads / $2016 / 04 /$ Reglamento $\% 20$ Organico\%20por\%20Procesos 1.pdf

[16] Universidad Laica Eloy Alfaro de Manabí (2016). Modelo Educativo. Retrieved from http://www. uleam.edu.ec/wp-content/uploads/2017/01/ ULEAM041-\%20Modelo\%20Educativo\%20 Uleam.pdf.

[17] Universidad Laica Eloy Alfaro de Manabí (2016). Plan Estratégico de Desarrollo Institucional. Retrieved from http://www.uleam.edu.ec/wpcontent/uploads/2018/05/PEDI-2016.-2020. ULEAM_.VERIFICADO2018.1.pdf.

[18] Universidad Laica Eloy Alfaro de Manabí (2016). Plan Institucional de Investigación 2016-2020. Retrieved from http://www. uleam.edu.ec/wp-content/uploads/2017/01/
PLAN\%20INSTITUCIONAL\%20DE\% 20 INVESTIGACION\%202016-2020.pdf.

[19] Universidad Laica Eloy Alfaro de Manabí (2016). Reglamento de Evaluación Integral del Desempeño del Personal Académico. Retrieved from http://www.uleam.edu.ec/wpcontent/uploads/2017/01/ULEAM015-\%20 REGLAMENTO-DE-EVALUACIONINTEGRAL-DEL-DESEMPENO-DELPERSONAL-ACADEMICO.pdf.Universidad Laica EloyAlfaro de Manabí (2016). Reglamento Reformatorio y Sustitutivo de Concurso Público de Méritos y Oposición para Docentes Titulares. Retrieved from http://www.uleam.edu.ec/ wp-content/uploads/2018/07/ULEAM019REGLAMENTO-REFORMATORIOY-SUSTITUTIVO-DE - CONCURSO M\%C3\%89RITO-Y-OPOSICI\%C3\%93N-CONLA-DEROGACI\%C3\%93N.pdf. 